УДК 621.383.4

\title{
АНАЛИЗ ЭФФЕКТИВНОСТИ ФОТОЭЛЕКТРИЧЕСКИХ СИСТЕМ КОММУНАЛЬНОГО НАЗНАЧЕНИЯ
}

\author{
Карпенко Д. С., Дубровская В.В., канд. техн. наук, Шкляр В.И., канд. техн. наук
}

Нацииональный технический университет Украины «Киевский политехнический институт», Институт энергосбережения и энергоменеджмента, ул. Борщзаговская, 115, Киев, 03056, Украина

Побудовано математичну модель роботи фотоелектричного модуля 3 водяною системою охолодження його поверхні. Проведено чисельне моделювання в програмному середовищі SolidWorks та визначено оптимальні конструктивні характеристики системи охолодження.
Построена математическая модель работы фотоэлектрического модуля с водяной системой охлаждения его поверхности. Проведено численное моделирование в программной среде SolidWorks и определены оптимальные конструктивные характеристики системы охлаждения.

Библ. 2, рис. 3.

A mathematical model of the solar module with a water cooling system of its surface was built. Numerical modeling with software SolidWorks and the optimal structural characteristics of the cooling system were determined.

Ключевые слова: математическая модель фотоэлектрического модуля, система охлаждения.

$G-$ расход воды, кг/с;

$I_{K 3}$ - ток короткого замыкания, А;

$k-$ постоянная Больцмана, Дж/К;

$n$ - коэффициент идеальности;

$P$ - уровень солнечной радиации на поверхности модуля, Вт;

$q$ - заряд электрона, Кл;

$S$ - расстояние между осями труб, м;

$T$ - температура, К;

$t_{\mathrm{CP}}-$ средняя температура поверхности, ${ }^{0} \mathrm{C}$;

Формирование конкурентных рынков электрической и тепловой энергии в Украине является одним из важнейших принципов существования эффективной энергетической отрасли страны, и, следовательно - ее экономики. Интегрирование солнечной энергетики в общую структуру энергетической системы Украины является актуальным вопросом на сегодняшний день, поскольку основным свойством данного источника энергии является его непостоянство во времени. Однако, при наличии инструментария по высокоточному прогнозированию генерации электрических и тепловых мощностей, солнечная энергетика могла бы быть одним из основных источников возобновляемой энергии в
$t_{\mathrm{MAX}}$ - максимальная температура поверхности, ${ }^{0} \mathrm{C}$;

$t_{\text {MIN }}$ - минимальная температура поверхности, ${ }^{0} \mathrm{C}$;

$U_{\mathrm{xx}}$ - напряжение холостого хода, В;

$\mathrm{u}_{\mathrm{oc}}-$ нормированное напряжение холостого хода, В;

$\Delta t$ - разница между максимальной и минимальной температурой поверхности, ${ }^{0} \mathrm{C}$;

КПД - коэффициент полезного действия;

ФЭП - фотоэлектрический преобразователь.

структуре энергобаланса страны.

Солнечный потенциал на территории Украины достаточен для его использования и преобразования в электрическую энергию с помощью фотоэлектрических установок. Учитывая уменьшение цены на фотоэлектрические системы и государственные механизмы стимуляции использования нетрадиционных источников энергии, фотоэлектрические установки становятся доступными для их использования в коммунальном хозяйстве.

В настоящее время львиная доля солнечных батарей имеют достаточно низкий КПД, который находится в диапазоне $10 . .20$ \%, поэтому вопрос о повышении эффективности использования 
энергии солнца является одним из важнейших в данной области альтернативной энергетики.

Эффективность солнечных элементов определяется коэффициентом полезного действия [1]: $\eta=\frac{f f \cdot I_{\mathrm{K} 3} \cdot U_{\mathrm{Xx}}}{P}$.

Коэффициент заполнения вольт-амперной характеристики рассчитывается по эмпирической формуле:

$f f=\frac{u_{\mathrm{OC}}-\ln \left(u_{\mathrm{OC}}+0,72\right)}{u_{\mathrm{OC}}+1}$,

где

$u_{\mathrm{OC}}=\frac{q}{n \cdot k \cdot T} \cdot U_{\mathrm{Xx}} \cdot$

Из уравнений видно, что при увеличении температуры солнечного элемента, снижается его коэффициент заполнения, а в результате и КПД. Критической температурой для кремниевых фотоэлементов является $85{ }^{\circ} \mathrm{C}$ [2]. Поэтому, возникает необходимость в охлаждении фотоэлемента для поддержания некоторой температуры полупроводника, при которой КПД будет максимальным, а при использовании полученной тепловой энергии повышается и общий КПД установки.

Цель работы. Определить оптимальные конструктивные характеристики системы охлаждения фотоэлементов.

Для решения поставленной задачи построена математическая модель фотоэлектрического модуля и проведено численное моделирование в программной среде SolidWorks.

Модель (рис. 1) состоит из четырех соединенных кремниевых монокристаллических фотоэлементов, под которыми установлен медный змеевик, с движущимся теплоносителем (вода) для снижения температуры солнечных элементов и получения дополнительного количества тепловой энергии.

Моделирование проводилось при следующих исходных данных: поступление солнечной радиации на поверхность модуля под прямым углом - 1000 Вт/м², скорость ветра над и под фотоэлементами - 3,5 м/с и температура окружающего воздуха $-15,2^{\circ} \mathrm{C}$. Плоскость модуля установлена параллельно горизонту. Характеристики монокристаллического кремниевого ФЭП К6М [2]: мощность 4...4,5 Вт, полный квадрат, размер в $156 \times 156 \pm 0,5$ мм, $f f=0,76$ и $\eta=18,46 \%$ при стандартных условиях.

Результаты моделирования работы фотоэлементов без охлаждения их поверхности приведены в табл. 1.
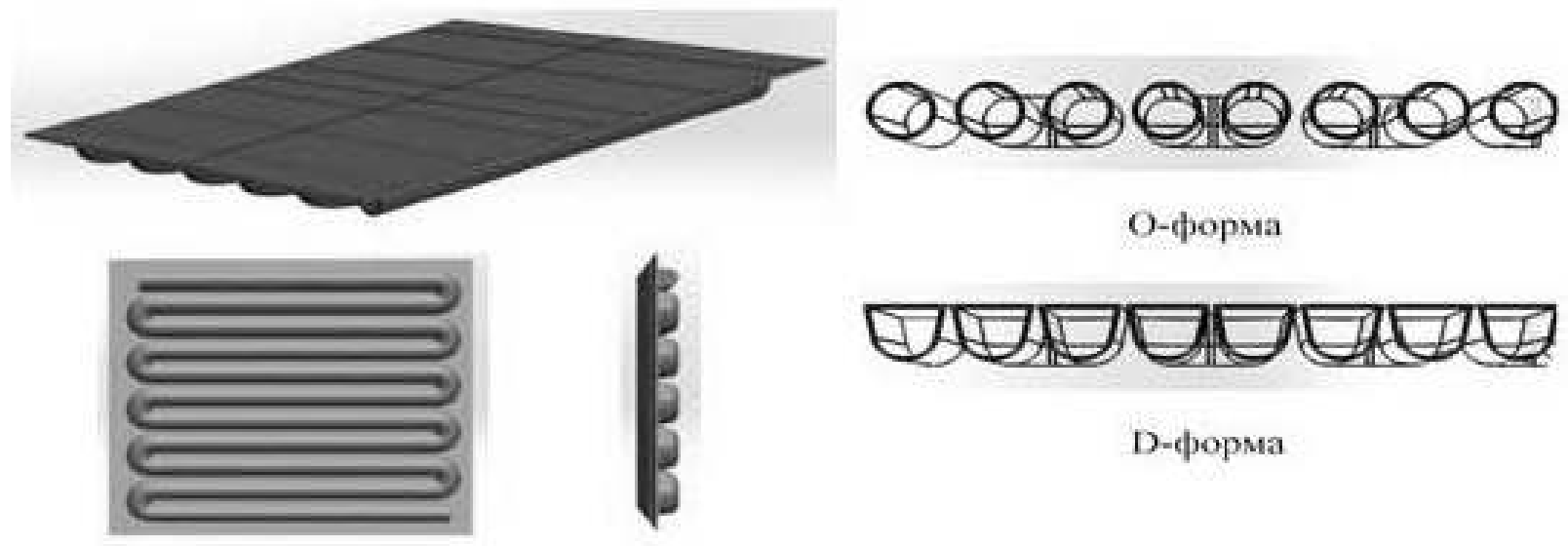

D-форма

Рис. 1. Модель фотоэлектрического модуля в программной среде SolidWorks 2014.

Таблица 1. Результаты моделирования без охлаждения фотоэлементов

\begin{tabular}{|c|c|c|c|c|}
\hline \multicolumn{2}{|c|}{ Температуры поверхности, ${ }^{\circ} \mathrm{C}$} & \multirow{2}{*}{$f f$} & \multirow{2}{*}{ КПД } \\
\hline$t_{\mathrm{CP}}$ & $t_{\mathrm{MAX}}$ & $t_{\mathrm{MIN}}$ & & \\
\hline 84,7 & 88,48 & 80,08 & 0,6335 & 0,1539 \\
\hline
\end{tabular}


По результатам моделирования видно, что средняя температура на поверхности фотоэлемента составляет $84,7^{\circ} \mathrm{C}$, что приводит к значительному снижению коэффициента заполнения $\mathrm{BAX}$ фотоэлементов, и в последствии к снижению КПД на $3 \%$.

Для получения минимально возможной температуры на поверхности ФЭП, при которой его КПД будет иметь максимальное значение, было проведено компьютерное моделирование с целью определения оптимальных конструктивных параметров системы охлаждения ФЭП: диаметр трубок змеевика, форма трубок и расстояние (шаг) между ними.

Для охлаждения ФЭП использовалась вода с температурой на входе в трубку $20{ }^{\circ} \mathrm{C}$ и массовыми расходами $\mathrm{G}=\{0,0025,0,01,0,025,0,1\}$ кг/с.

Внутренние диаметры трубок змеевика составляли $\mathrm{d}=\{10 ; 15,20\}$ мM, а шаг равнялся внутреннему диаметру трубы. Были рассмотрены два вида форм трубок: O-формы и D-формы.

Результаты моделирования системы охлаждения поверхности ФЭП с трубками О-формы с различными диаметрами приведены в табл. 2.
На основании результатов можно сделать вывод, что наименьшая средняя температура и равномерность охлаждения поверхности фотоэлементов, которая характеризуется разницей между максимальной и минимальной температурой поверхности, наблюдается при внутреннем диаметре трубки змеевика 20 мм. Дальнейшее увеличение диаметра приводит к возрастанию расхода воды и энергетических затрат на ее циркуляцию.

Результаты моделирования системы охлаждения ФЭП с трубками змеевика D-формы при тех же условиях проведения эксперимента при эквивалентном диаметре трубок 20 мм приведены в табл. 3, а их сравнение с трубками змеевика О-формы на рис. 2, $a$. Из рисунка видно, что наименьшая средняя температура и равномерность охлаждения поверхности фотоэлементов прослеживается при D-форме трубки змеевика. Следовательно, в дальнейшем использовалась трубка D-формы, как более эффективный вариант.

Результаты моделирования при изменении расстояния между осями труб в интервале $S=20 \ldots 40$ мм приведены в табл. 3 и на рис. 2,6 .

Таблица 2. Результаты моделирования системы охлаждения поверхности ФЭП с трубками О-формы

\begin{tabular}{|c|c|c|c|c|c|c|c|}
\hline \multirow{2}{*}{$\begin{array}{c}\text { Внутренний } \\
\text { диаметр } \\
\text { трубки, мм }\end{array}$} & \multirow{2}{*}{$\begin{array}{c}\text { Расход воды, } \\
\text { кг/с } \\
G \\
\end{array}$} & \multicolumn{4}{|c|}{ Температуры поверхности, ${ }^{\circ} \mathrm{C}$} & \multirow{2}{*}{$f f$} & \multirow{2}{*}{ КПД } \\
\hline & & $t_{\mathrm{CP}}$ & $t_{\mathrm{MAX}}$ & $t_{\mathrm{MIN}}$ & $\Delta t$ & & \\
\hline \multirow{4}{*}{10} & 0,0025 & 40,08 & 63,28 & 25,36 & 37,92 & 0,7238 & 0,1758 \\
\hline & 0,01 & 34,34 & 52,64 & 23,03 & 29,61 & 0,7373 & 0,1791 \\
\hline & 0,025 & 31,82 & 49,08 & 22,13 & 26,95 & 0,7434 & 0,1806 \\
\hline & 0,1 & 27,45 & 43,52 & 20,83 & 22,69 & 0,7542 & 0,1832 \\
\hline \multirow{4}{*}{15} & 0,0025 & 38,11 & 58,62 & 25,73 & 32,89 & 0,7284 & 0,1769 \\
\hline & 0,01 & 32 & 47,24 & 23,23 & 24,01 & 0,7430 & 0,1805 \\
\hline & 0,025 & 29,77 & 44,75 & 22,17 & 22,58 & 0,7484 & 0,1818 \\
\hline & 0,1 & 25,85 & 38,68 & 20,91 & 17,77 & 0,7583 & 0,1842 \\
\hline \multirow{4}{*}{20} & 0,0025 & 37,95 & 56,46 & 25,44 & 31,02 & 0,7287 & 0,1770 \\
\hline & 0,01 & 31 & 44,7 & 23,19 & 21,51 & 0,7454 & 0,1810 \\
\hline & 0,025 & 29,12 & 41,7 & 22,28 & 19,42 & 0,7500 & 0,1822 \\
\hline & 0,1 & 25,62 & 38,57 & 21,29 & 17,28 & 0,7563 & 0,1837 \\
\hline
\end{tabular}


Таблица 3. Результаты моделирования системы охлаждения ФЭП с трубками D-формы и различным шагом

\begin{tabular}{|c|c|c|c|c|c|c|c|}
\hline \multirow{2}{*}{$\begin{array}{c}\text { Внутренний } \\
\text { диаметр } \\
\text { трубки, мм }\end{array}$} & \multirow{2}{*}{$\begin{array}{c}\text { Расход } \\
\text { воды, } \\
\text { кг/с } \\
G\end{array}$} & \multicolumn{4}{|c|}{ Температуры поверхности, ${ }^{\circ} \mathrm{C}$} & \multirow[t]{2}{*}{$f f$} & \multirow[t]{2}{*}{ КПД } \\
\hline & & $t_{\mathrm{CP}}$ & $t_{\mathrm{MAX}}$ & $t_{\mathrm{MIN}}$ & $\Delta t$ & & \\
\hline \multicolumn{8}{|c|}{$S=40 \mathrm{MM}$} \\
\hline \multirow{4}{*}{20} & 0,0025 & 32,55 & 48,11 & 24,34 & 23,77 & 0,7416 & 0,1801 \\
\hline & 0,01 & 25,64 & 35,4 & 22,62 & 12,78 & 0,7588 & 0,1843 \\
\hline & 0,025 & 23,89 & 32,55 & 21,82 & 10,73 & 0,7633 & 0,1854 \\
\hline & 0,1 & 21,97 & 29,73 & 21,01 & 8,72 & 0,7682 & 0,1866 \\
\hline \multicolumn{8}{|c|}{$S=26$ мм } \\
\hline \multirow{4}{*}{20} & 0,0025 & 31,13 & 45,76 & 23,62 & 22,14 & 0,7451 & 0,1810 \\
\hline & 0,01 & 26,57 & 37,65 & 22,61 & 15,04 & 0,7564 & 0,1837 \\
\hline & 0,025 & 22,56 & 30,54 & 21,32 & 9,22 & 0,7667 & 0,1862 \\
\hline & 0,1 & 21,8 & 29,3 & 21 & 8,3 & 0,7687 & 0,1867 \\
\hline
\end{tabular}

Исходя из результатов моделирования установлено, что наименьшая средняя температура и равномерность охлаждения поверхности фотоэлементов отмечена при $S=26$ мм. Следовательно, при дальнейшем анализе конструк- тивных характеристик охлаждения экспериментальной установки, использовали змеевик с расстоянием между осями трубок $S=26$ мм, как оптимальный вариант.

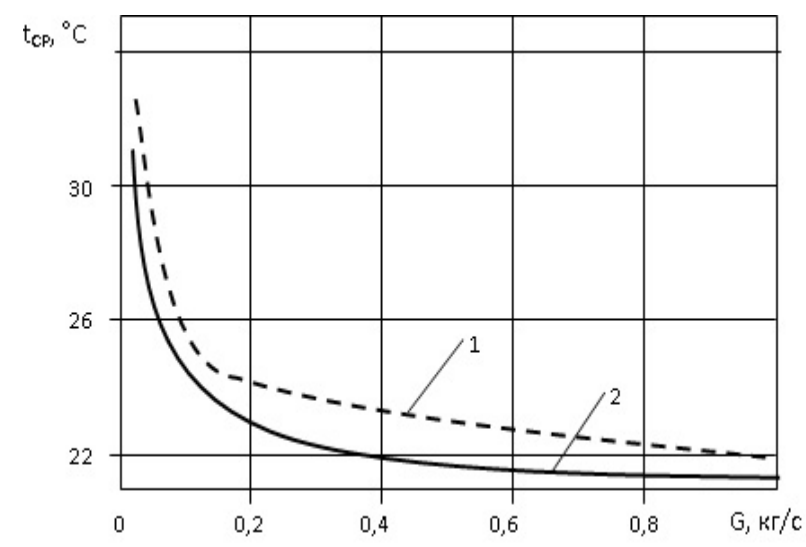

6)

Рис. 2. Зависимость средней температуры поверхности от расхода воды в змеевике: а) различные формы трубок: 1 - О-форма; 2 -D-форма; б) различные чиги между осями труб: $1-S=40$ мм; $2-S=26$ мм.

На основе результатов моделирования построен график зависимости вырабатываемой электрической мощности от расхода охлаждаемой воды (рис. 3). 


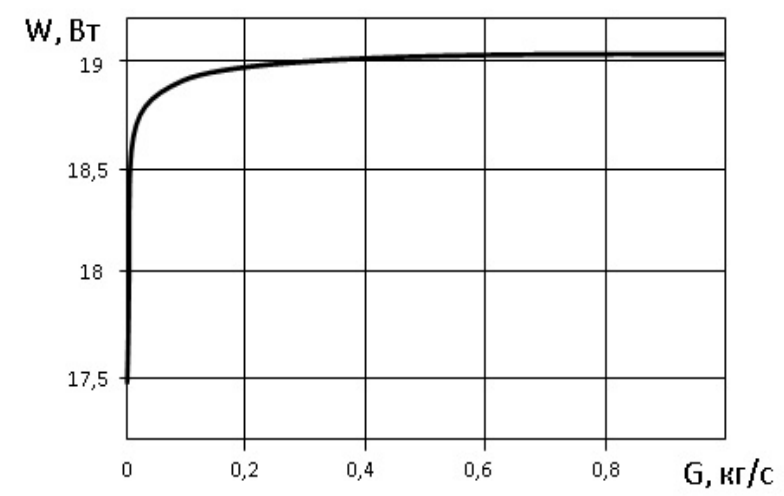

Рис. 3. Зависимость вырабатываемой электрической мощности от расхода воды.

\section{Bыводы}

1. Выполнено численное моделирование работы ФЭП без охлаждения и с охлаждением его поверхности в программном обеспечении SolidWorks 2014.

2. Исследованы зависимости температуры на поверхности фотоэлементов от расхода воды, протекающей в змеевике.

3. Проанализированы результаты моделирования и выбраны оптимальные конструктивные характеристики для охлаждения фотоэлементов: трубки D-формы с эквивалентным диаметром

\section{ANALYSIS OF EFFICIENCY PHOTOVOLTAIC SYSTEMS MUNICIPAL PURPOSE}

\section{Karpenko D. S., Dubrovska V.V., Shklyar V.I.}

National Technical University of Ukraine "Kiev Polytechnic Institute", Institute of Energy Saving and Energy Management, vul. Borshchagivska, 115, Kyiv, 03056, Ukraine.

A mathematical model of the solar module with a water cooling system of its surface was built. Numerical modeling with software SolidWorks and the
20 мм, шагом 26 мм и расходом воды около $0,2 \mathrm{\kappa} / \mathrm{c}$.

4. Охлаждение ФЭП позволило увеличить его КПД на 3,3 \% по сравнению с неохлаждаемым и производство электроэнергии с 17,5 Вт до 19 Вт.

\section{ЛИТЕРАТУРА}

1. Photovoltaic Education Network: [Электронный ресурс]. - Режим доступа: http://pveducation. org.

2. KVAZAR // [Электронный ресурс]. - Режим доступу: http://www.kvazar.com.

optimal structural characteristics (diameter and form tubes wheelbase coil tubes) of the cooling system were determined. The dependence of the generated electrical power from rate cooling water by the solar module was received.

References 2, tables 3, figures 3 .

Key words: mathematical model of the solar module, the cooling system.

1. Photovoltaic Education Network: http:// pveducation.org.

2. KVAZAR: http://www.kvazar.com.

Получено 08.02.2016

Received 08.02.2016 